\title{
Aggressive Vertebral Hemangioma Causing Acute Spinal Cord Compression
}

\author{
Sokol Trungu ${ }^{1,2}$ Stefano Forcato ${ }^{1,2}$ Antonio Scollato ${ }^{1}$ Massimo Miscusi ${ }^{2}$ Antonino Raco ${ }^{2}$
}

${ }^{1}$ Neurosurgery Unit, Card. G. Panico Hospital, Tricase, Italy

2Department of Neuroscience, Mental Health, and Sense Organs, Faculty of Medicine and Psychology, "Sapienza" University of Rome, Rome, Italy

| Neurosci Rural Pract 2019;10:672-674

A 46-year-old woman presented to our emergency department with sudden onset of lower extremity weakness after physical activity. She referred only dorsal back pain before these symptoms. Neurologic examination revealed weakness $(2 / 5)$ of lower limbs, hyperreflexia of deep tendon reflex of lower limbs, hypoesthesia under D7 level, and no sphincteric dysfunction. A computed tomography scan showed an
Address for correspondence Sokol Trungu, MD, Via Pio X 4 Tricase, Italy (e-mail: s_trungu@hotmail.com).

accentuation of trabecular markings within the vertebral body and areas of lysis ( - Figs. 1A and $\mathbf{F}$ ). Contrast-enhanced magnetic resonance images show diffuse abnormal marrow signal throughout the T6 vertebral body with epidural components with spinal cord compression ( - Fig. 1B-H).

She underwent surgery on the same day through a mini-open decompression and percutaneous short posterior

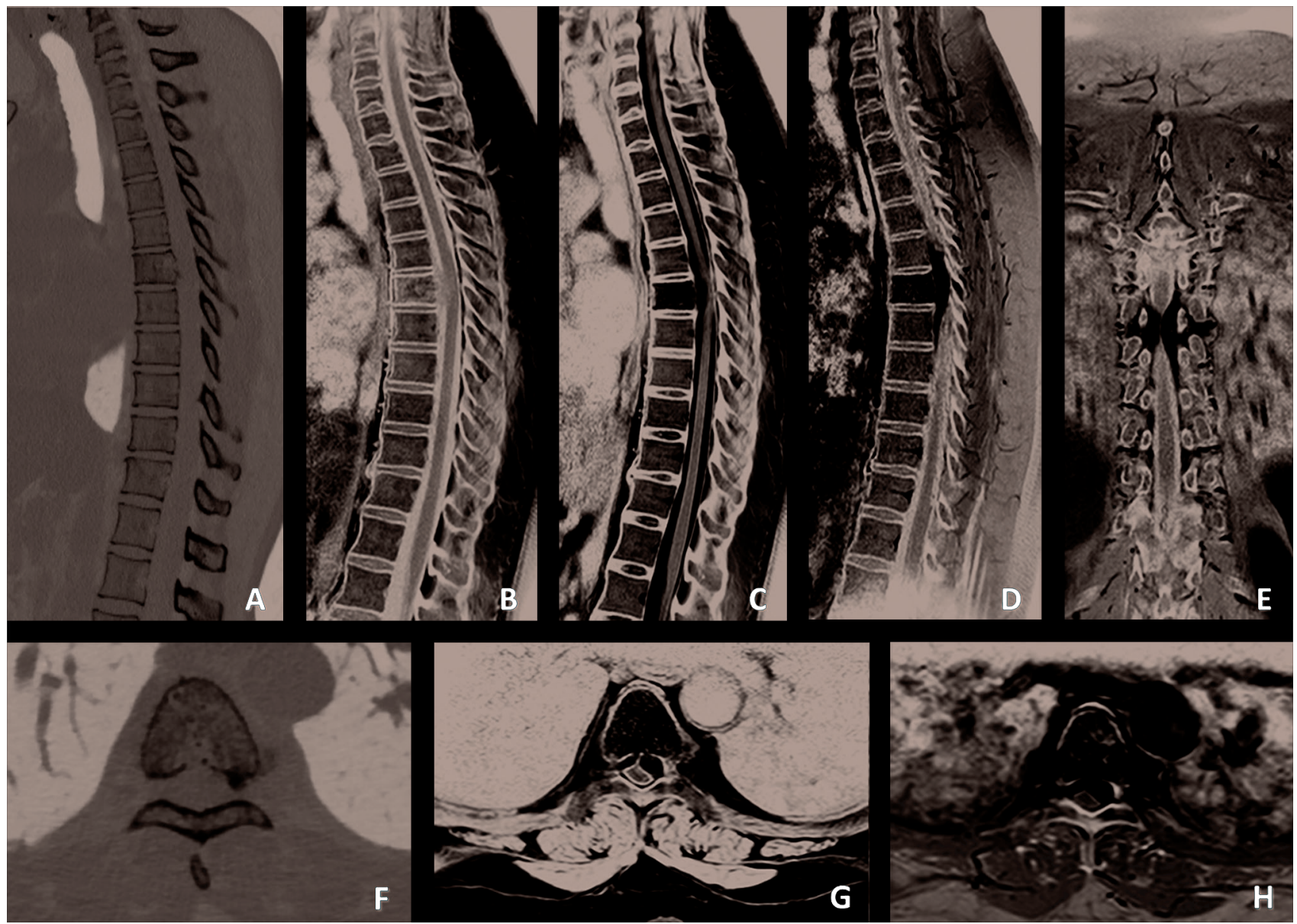

Fig. 1 Sagittal (A) and axial (F) computed tomography images demonstrating accentuation of trabecular markings within the vertebral body and areas of lysis involving the entire T6 vertebral body. Sagittal T1-weighted (B); sagittal (C) and axial (G) T2-weighted; sagittal (D), coronal $(\mathbf{E})$, and axial $(\mathbf{H})$ contrast-enhanced magnetic resonance images showing a T6 aggressive hemangioma with epidural extension and severe anterior cord compression.

(c)2019 Association for Helping Neurosurgical Sick People
License terms

(요 (1) $\Theta \circledast$ 


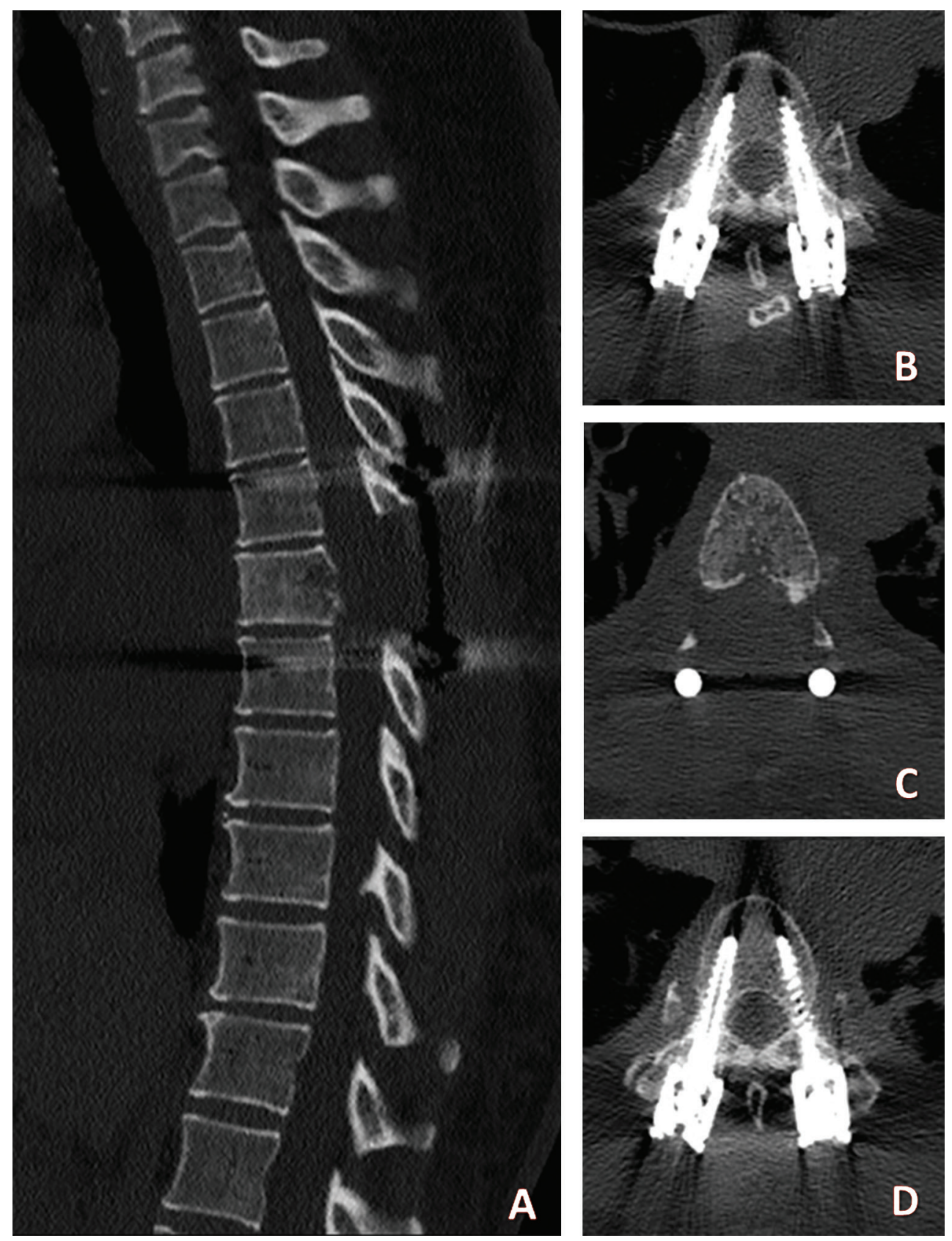

Fig. 2 Sagittal (A) and axial (B-D) postoperative computed tomography images demonstrating the posterior decompression and short pedicle screw fixation.

fixation (-Fig. 2). No complications occurred after surgery with full recovery of neurological symptoms. Radiotherapy was perfomed after 4 weeks with resolution of dorsal back pain.

Vertebral hemangiomas $(\mathrm{VH})$ are benign and generally asymptomatic primary vascular tumors of bone., ${ }^{1,2}$ Rarely, these lesions can cause symptoms due to cord compression as a result of bone expansion, erosion through cortex, fracture, or hematoma. ${ }^{3}$ Despite our long-standing recognition of aggressive $\mathrm{VH}$, there is still a controversy regarding the optimal treatment strategy, and numerous therapeutic options have been described: embolization, surgery, radiotherapy, vertebroplasty, or a combination of them. ${ }^{4-9}$

\section{Funding}

None.

\section{Conflict of Interest}

None declared.

\section{References}

1 Enneking WF. A system of staging musculoskeletal neoplasms. Clin Orthop Relat Res 1986; ( 204):9-24

2 Friedman DP. Symptomatic vertebral hemangiomas: MR findings. AJR Am J Roentgenol 1996;167(2):359-364

3 Goldstein CL, Varga PP, Gokaslan ZL, et al. Spinal hemangiomas: results of surgical management for local recurrence and mortality in a multicenter study. Spine 2015;40(9):656-664 
4 Guarnieri G, Ambrosanio G, Vassallo P, et al. Vertebroplasty as treatment of aggressive and symptomatic vertebral hemangiomas: up to 4 years of follow-up. Neuroradiology 2009;51(7):471-476

5 Jiang L, Liu XG, Yuan HS, et al. Diagnosis and treatment of vertebral hemangiomas with neurologic deficit: a report of 29 cases and literature review. Spine J 2014;14(6):944-954

6 Urrutia J, Postigo R, Larrondo R, Martin AS. Clinical and imaging findings in patients with aggressive spinal hemangioma requiring surgical treatment. J Clin Neurosci 2011;18(2):209-212
7 Raco A, Ciappetta P, Artico M, Salvati M, Guidetti G, Guglielmi G. Vertebral hemangiomas with cord compression: the role of embolization in five cases. Surg Neurol 1990;34(3):164-168

8 Smith TP, Koci T, Mehringer CM, et al. Transarterial embolization of vertebral hemangioma. J Vasc Interv Radiol 1993;4(5):681-685

9 Heyd R, Seegenschmiedt MH, Rades D, et al; German Cooperative Group on Radiotherapy for Benign Diseases. Radiotherapy for symptomatic vertebral hemangiomas: results of a multicenter study and literature review. Int J Radiat Oncol Biol Phys 2010;77(1):217-225 